\title{
Joint Optimization of Switching Threshold and Power Allocation in One Way Incremental Amplify and Forward Cooperative Networks
}

\author{
Ines Hosni ${ }^{1}$, Noureddine Hamdi ${ }^{2}$ \\ Université de Tunis El Manar, École Nationale d'Ingénieurs de Tunis, \\ LR-99-ES21 Laboratoire de Systèmes de Communications, 1002, Tunis, Tunisie; \\ 1 ines.hosni@hotmail.fr \\ 2 noureddine.hamdi@ept.rnu.tn
}

\begin{abstract}
Incremental adaptive relaying has shown an attractive compromise between robustness and spectral efficiency in cooperative networks. Relays are used to incrementally assist sources to transmit data to their destinations. In this paper, a joint optimization of switching thresholds and transmit power assignments is proposed. The relaying is used, if either the signal strength at the destination is lower than a given threshold and at the relay is respectively and greater than another threshold. In the relaying phase, optimal transmit power assignment is considered at the source and at the relay. The proposed scheme permits to meet the best compromise among reliability, throughput and energy efficiency of cooperative source -relay- destination transmissions. We apply the proposed scheme to amplify and forward (AF) relaying systems. A selection of numerical results is illustrated for the performance analysis.
\end{abstract}

\section{INTRODUCTION}

Next generations of wireless packet access networks have to provide a large range of new data and multimedia services at low costs, high reliability and quality of service (QoS) requirements. However, the available radio spectrum is used intensively, energy consumption is limited and the wireless channel is dispersive by nature [1]. Consequently, meet the increasing expectations in throughput of wireless communications remains open for future research. In fact, several advanced features have been adopted for updating existing networks and development for future networks [2].

In interference limited networks, the desired coverage and throughput cannot be achieved by direct point-to-point transmission in either infrastructure-based or ad hoc networks. Cooperative transmissions [3] by considering relays to assist sources to send data to destinations, have now emerged as an attractive approach to meet these goals. The idea is to reduce the distance between a source and a destination node by using several hops and overcome the shadowing attenuations. Furthermore, the non linear relationship between the source and destination helps in reducing end-to-end attenuation, path-loss and thus in relaxing the use of parameters of link budgets. The two popular techniques of cooperative diversity are the Amplify-and-Forward(AF)[4] which amplifies the signal before transmitting and the Decode-and-Forward (DF)scheme which demodulates - decodes the signal before transmission[5]. In [6] and [7], the authors drift closedform expressions for the outage probability for respectively Rayleigh and Nakagami-m channels with decode-and-forward relays. In this work we are interested by the Rayleigh channel model with amplify-and-forward relay.

The AF scheme is considered as a reduced complexity relaying strategy, which is due to the alleviated processing at the relays by directly forwarding signals to the destination. The joint optimization of switching threshold and power allocation have not received sufficient interests in related literature.In this paper we contributed in providing solutions for the best switching threshold between direct and relayed transmissions and the optimal power allocation to both the source and the relay. According to our knowledge there are not answers in the literature to the last problem.

The remainder of this paper is organized in three sections. In section II we present the system model,in section III we present an analytical approach of the expressions for the error probability, and average channel capacity of the AF incremental relaying system. Section IV exposes the numerical results and performance analysis.

\section{System Model}

We consider a network model consisting of three nodes: a source $(S)$, a relay $(R)$, and a destination $(D)$ as shown in Fig. 1. It is assumed that each node is equipped with a single antenna. Additive white Gaussian noise (AWGN) terms at all links are assumed to have zero mean and equal variances $\sigma_{n}^{2}=N_{0} / 2$.

We denote the source-destination, source-relay and relaydestination links by S-D, S-R, and R-D, respectively. In what follows, signals on these links are modeled with Rayleigh fading. The corresponding channel gains are $h_{S D}, h_{S R}$, and $h_{R D}$.

We assume that the total transmission power $P_{t}$ is limited and shared by nodes $S$ and $R$, by power allocation $\nu P_{t}$ to $S$ and $(1-\nu) P_{t}$ to $R$, where $\nu$ is the cooperative power ratio. 


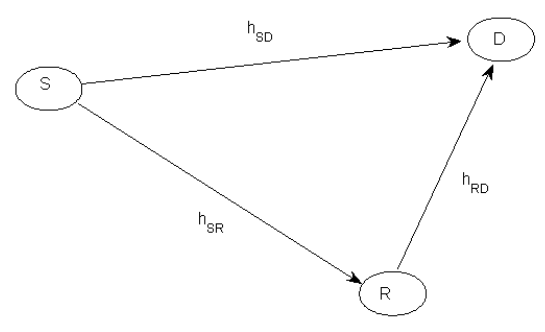

Fig. 1. System Model

The distances between node pairs are: $d$ for $S-D\left(d_{S D}\right)$, and approximated by $r d$ and $(1-r) d$ for $S-R$ and $R-D$ respectively $\left(d_{S R}\right.$ and $\left.d_{R D}\right)$, where $0 \leq r \leq 1$ is a normalized distance defined as a ratio between the distances $S-R$ and $S-D$.

Let $\rho=\frac{p_{t}}{N_{0}}$ be the total power to noise ratio. Then,the instantaneous SNR $\gamma_{i j}=\nu_{i j} \rho|h i j|^{2}$, where $\nu_{i j}$ is the power ratio used in link $i-j,\left(\nu_{i j}=1\right.$ for conventional direct link $)$.

The model is based on two phases:

- In the first phase,the source broadcasts data to nodes $\mathrm{R}$ and $\mathrm{D}$ which receive noisy versions of the source signal. The destination, based on strength of the received signal, decides if the relay is required to assist it or not.

- In the second phase, if the signal strength at node D is greater than a threshold, the relay does nothing and the source sends a new message. If the signal strength is low at the destination, the node $\mathrm{R}$ forwards the received signal, if its strength is greater than a threshold to the destination. In this case,the destination combines the two received signals via a Maximum Ratio Combining strategy(MRC)to obtain combined signal Yr.

- Otherwise the relay remains idle and the destination requests another copy from the source.

We assume that measuring the signal quality at the destination is based on the comparison of the received SNR of the source with a SNR threshold $\gamma_{s}$, which identifies the minimum for which the destination can detect the signal without the necessity of the relay.

At the first phase, $\left(Y_{S D}\right)$ and $\left(Y_{S R}\right)$ are the received signals at the destination and at the relay:

$$
\begin{aligned}
& Y_{S D}=\sqrt{P_{t}} h_{S D} x+n_{S D} \\
& Y_{S R}=\sqrt{P_{t}} h_{S R} x+n_{S R}
\end{aligned}
$$

where,

$x$ : The transmitted symbol.

$h_{S D}$ : The channel coefficient for the link between the source $\mathrm{S}$ and the destination $\mathrm{D}$.

$h_{S R}$ : The channel coefficient for the link between the source $\mathrm{S}$ and the relay $\mathrm{R}$.

$n_{S D}$ and $n_{S R}$ : Additive complex Gaussian noise AWGN with a variance equal to $\sigma_{n}$.

In AF incremental relaying system, the relayed signal $Y_{R D}$ is an amplified copy of $Y_{S R}$.

In fact, the expression of signal from the R-D link can be written as:

$$
Y_{R D}=h_{R D} Y A_{S R}+n_{R D}
$$

Where, $Y A_{S R}$ is the signal amplified by the relay given by:

$$
Y A_{S R}=\frac{Y_{S R}}{E\left(\sqrt{\left|h_{S R} x+n_{S R}\right|^{2}}\right)}
$$

$$
Y A_{S R}=\frac{Y_{S R}}{\sqrt{P_{t}\left|h_{S R}\right|^{2}+\sigma_{n}^{2}}}
$$

$\mathrm{E}($.$) is the statistical average operator.$

We define the amplification factor given by:

$$
G=\frac{1}{\sqrt{P_{t} h_{S R}^{2}+\sigma_{n}^{2}}}
$$

So,we can represent the received signal of the R-D link as follows:

$$
Y_{R D}=h_{R D} G Y_{S R}+n_{R D}
$$

The combined signal $\mathrm{Yr}$ obtained at the output of the destination Maximum Ratio Combiner (MRC) is :

$$
Y r=\frac{\sqrt{P_{t}}}{\sigma_{n}^{2}} Y_{S D} h_{S D}^{*}+\frac{Y_{R D} h_{R D}^{*} h_{S R}^{*} G \sqrt{P_{t}}}{\sigma_{n}^{2}\left(1+G^{4} h_{R D}^{2}\right)}
$$

The SNR at the output of the MRC at the destination can be represented as:

$$
\gamma_{M R C}=\gamma_{S D}+\frac{\gamma_{S R} \gamma_{R D}}{1+\gamma_{S R}+\gamma_{R D}}
$$

where, $\gamma_{S I}=\frac{P_{t}}{\sigma_{n}^{2}}\left|h_{S I}\right|^{2}, I=R$ or $D$ is the SNR for the relaying link between the source $S$ and the relay $R$ or the the destination D, and $\gamma_{R D}=\frac{P_{t}}{\sigma_{n}^{2}}\left|h_{R D}\right|^{2}$ is the SNR for the relaying link between the relay $R$ and the destination $D$.

Using the average SNR $\gamma_{\bar{S} R}=E\left(h_{S R}^{2}\right) \frac{P_{t}}{N_{0}}$ and $\gamma_{\bar{R} D}=E\left(h_{R D}^{2}\right) \frac{P_{t}}{N_{0}}$ we can express the pdf of $\gamma_{s}$ as: $f_{\gamma_{s}}(\gamma)=\frac{1}{\overline{\gamma_{s}}} \exp \left(\frac{-\gamma}{\overline{\gamma_{s}}}\right)$, where $\overline{\gamma_{s}}=\frac{\gamma_{\bar{S} R} \bar{R}_{\bar{R}}}{\gamma_{\bar{S} R}+\gamma_{\bar{R} D}}$

To simplify the performance analysis, we can approximate the total SNR by its upper bound:

$$
\begin{aligned}
& \gamma_{M R C} \leq \gamma_{S D}+\min \left(\gamma_{S R}, \gamma_{R D}\right) \\
& \gamma_{M R C} \leq \gamma_{S D}+\gamma_{s}
\end{aligned}
$$

In order to capture the path-loss effect on the performance,we use these average expressions where, $E\left(h_{S R}^{2}\right)=$ $\left(\frac{d_{S D}}{d_{S R}}\right)^{\alpha} E\left(h_{R D}^{2}\right)=\left(\frac{d_{S D}}{d_{R D}}\right)^{\alpha}$ and, $E\left(h_{S D}^{2}\right)=1$

We denote that $\left(d_{S D}\right)^{\alpha}=k d_{0}^{\alpha}$.

$\alpha$ is the path-loss exponent.

$k=P_{t} * k_{0}$,where $k_{0}$ is a constant.

\section{THEORICAL ANALYSIS}

Here, we are going to derive the error probability (BER), the capacity $(\mathrm{C})$ of the AF incremental relaying protocol. In this study, we consider the linear network as shown in Fig.2. 
Fig. 2. Direct link

The considered threshold $s$ is used to switch between direct transmission and cooperation. It is assumed that, if the condition $\gamma_{S D}>\gamma_{s}$ is satisfied, the destination can decode the received signal with less error. $\gamma_{s}$ is defined in [8] and given by:

$$
\gamma_{s}=\frac{-2}{3} \ln \left(5 B E R_{0}\right)\left(2^{n}-1\right)
$$

where $n$ is the number of bits per symbol .

Then, the SNR of the link S-D $\gamma_{S D}$ is statistically modelled by an exponential distribution and its CDF is given by:

$$
\operatorname{prob}\left(\gamma_{S D} \leq \gamma_{s}\right)=1-\exp \left(\frac{-\gamma_{s}}{\gamma_{\bar{S} D}}\right)
$$

where $\gamma_{S} D=E\left(\gamma_{S D}\right)=\frac{P_{t} P_{L S D}}{\sigma^{2}}$ is the average SNR between $\mathrm{S}$ and $\mathrm{D}, P_{L i j}=k_{0} d_{i j}^{-\alpha}$ is the pathloss defined between nodes $\mathrm{i}$ and $\mathrm{j}$.

\section{A. Capacity Characteristics}

In amplify and forward incremental relaying system, the destination compares first the received SNR to a preset threshold. If the SNR at the destination is greater than the SNR threshold, it decodes the received signal from the direct link without requesting the relay cooperation.

Then, if $\gamma_{S D}>\gamma_{s}$ direct link is used.

In several cases, the distribution of power between the different transmitters can improve the capacity whose expression is:

$$
C=\log \left(1+\gamma_{S D}\right)
$$

On the other hand, if the SNR is smaller than the threshold, the relay is requested to forward the received signal from the source to its destination, for diversity combining.

Then, if $\gamma_{S D} \leq \gamma_{s}$ relaying link, the capacity can be expressed by:

$$
\begin{gathered}
C=\frac{1}{2} \log \left(1+\gamma_{M R C}\right) \\
C=\frac{1}{2} \log \left(1+\gamma_{S D}+\frac{\gamma_{S R} \gamma_{R D}}{1+\gamma_{S R}+\gamma_{R D}}\right)
\end{gathered}
$$

\section{B. Error probability analysis}

For the direct link, and if M-QAM is the modulator, the expression of the BEP can be written as:

$$
P_{e S D} \approx b Q\left(\sqrt{a \gamma_{S D}}\right)
$$

Where

$\mathrm{Q}(\mathrm{x})$ : is the Gaussian Q-function defined as:

$$
Q(x)=\frac{1}{\sqrt{2 \pi}} \int_{x}^{\infty} \exp \left(-\frac{x^{2}}{2}\right) d x
$$

$P_{e S D}$ given by equation (15) is a strict equality if the modulator is BPSK or QPSK.In this case, we have $b=1$ and $a=0.5$. So, $P_{e S D}$ becomes:

$$
P_{e S D}=Q\left(\sqrt{\frac{1}{2} \gamma_{S D}}\right)
$$

The average error probability between $\mathrm{S}$ and $\mathrm{D}$ can be written as :

$$
P(e)=\operatorname{Pr}\left(\left|\gamma_{S D}\right|^{2} \leq \gamma_{s}\right) P_{e_{S R D}}+\operatorname{Pr}\left(\left|\gamma_{S D}\right|^{2}>\gamma_{s}\right) P_{e_{\text {direct }}}
$$

$$
P(e)=\left(1-\exp \left(\frac{-\gamma_{s}}{\gamma_{\bar{S} D}}\right)\right) P_{e_{S R D}}+\exp \left(\frac{-\gamma_{s}}{\gamma_{\bar{S} D}}\right) P_{e_{\text {direct }}}
$$

where, $P_{e_{\text {direct }}}$ is the error probability between the source and the destination. $P_{e_{S R D}}$ is the probability of error for the relaying link.

The error probability can be written as : $a \times \operatorname{erf} c\left(\sqrt{b \gamma_{S D}}\right)$ where, $\operatorname{erfc}(x)=\frac{2}{\sqrt{\pi}} \int_{x}^{\infty} \exp \left(-x^{2}\right) d x$.

The parameters $\mathrm{a}$ and $\mathrm{b}$ depend on the modulation type (for BPSK: $a=0.5$ and $b=1$ )

Then, the error probability of direct link can be written as :

$$
P_{e_{\text {direct }}}(e)=\int_{0}^{\infty} P_{e_{\text {direct }}}(e \mid \gamma) f_{\gamma_{S D} \mid \gamma_{S D}>\gamma_{s}}(\gamma) d \gamma
$$

where, $f_{\gamma_{S D} \mid \gamma_{S D}>\gamma_{s}}(\gamma)$ is the conditional probability density function (PDF) of $\gamma_{S D}$. For $\gamma>\gamma_{s}$ it can be found to be as:

$$
f_{\gamma_{S D} \mid \gamma_{S D}>\gamma_{s}}(\gamma)=\frac{\exp \left(\frac{\gamma_{s}}{\gamma_{\bar{S} D}}\right)}{\gamma_{\bar{S} D}} \exp \left(\frac{-\gamma}{\gamma_{\bar{S} D}}\right)
$$

From (20) and (21),the average error probability can be written as:

$$
\begin{array}{r}
P_{e_{\text {direct }}}(e)=\operatorname{aerfc}\left(\sqrt{b \gamma_{s}}\right)-\operatorname{aexp}\left(\frac{\gamma_{s}}{\gamma_{\bar{S} D}}\right) \\
\times \sqrt{\frac{b \gamma_{\bar{S} D}}{1+b \gamma_{\bar{S} D}}} \operatorname{erfc}\left(\sqrt{\gamma_{s}\left(b+\frac{1}{\gamma_{\bar{S} D}}\right.}\right)
\end{array}
$$

We note that when $\gamma_{s}$ increases, $P_{e_{\text {direct }}}(e)$ decreases, which affects the value of $P_{e}$ for a high SNR. Receiving a second copy of the signal from the relay, the destination combines the two signals. The error probability of the link using relay is expressed as follows:

$$
P_{e_{S R D}}(e)=a \int_{0}^{\infty} f_{\gamma_{v} \mid \gamma_{S D} \leq \gamma_{s}}\left(\gamma_{v}\right) \operatorname{erf} c\left(\sqrt{b \gamma_{v}}\right) d \gamma_{v}
$$

The conditional PDF $f_{\gamma_{v} \mid \gamma_{S D} \leq \gamma_{s}}\left(\gamma_{v}\right)$ is determined by the convolution of the PDF of $\gamma_{S D}$ and $\gamma_{s}$.

Where, the pdf of $\gamma_{S D}$ is given by:

$$
f_{\gamma S D}(\gamma)=\frac{1}{\gamma_{\bar{S} D}} \exp \left(\frac{-\gamma}{\gamma_{\bar{S} D}}\right)
$$

and the pdf of $\gamma_{s}$ can be written as:

$$
f_{\gamma_{s}}(\gamma)=\frac{1}{\gamma_{\bar{S} D}} \exp \left(\frac{-\gamma}{\overline{\gamma_{s}}}\right)
$$


So, the conditional probability density function can be found to be as:

$$
f_{\gamma_{v} \mid \gamma_{S D} \leq \gamma_{s}}(\gamma)=\frac{1}{\bar{\gamma}_{S D}} \exp \left(\frac{\gamma_{s}}{\bar{\gamma}_{S D}}\right) \exp \left(\frac{-\gamma}{\bar{\gamma}_{S D}}\right)
$$

From (23) and (26),the average error probability can be written as:

$$
P_{e_{S R D}}(e)=a \int_{0}^{\infty} \frac{1}{\bar{\gamma}_{S D}} \exp \left(\frac{\gamma_{s}}{\bar{\gamma}_{S D}}\right) \exp \left(\frac{-\gamma}{\bar{\gamma}_{S D}}\right) \operatorname{erfc}(\sqrt{b \gamma}) d \gamma
$$

\section{NUMERICAL RESULTS AND PERFORMANCE ANALYSIS}

The parameters used for the presented numerical results are:

Distance between the source $\mathrm{S}$ and the destination $\mathrm{D}$ is $d=10 \mathrm{Km}$.

We consider the path-loss exponent $\alpha=3$. The distances between the three nodes as follows: $d_{S D}=d, d_{S R}=r d$ and $d_{R D}=(1-r) d$.

$\bar{\gamma}_{i j}=k * d_{i j}^{-\alpha}$ where (i=S or $\left.\mathrm{i}=\mathrm{R}\right)$ and $(\mathrm{j}=\mathrm{R}$ or $\mathrm{j}=\mathrm{D})$.

The power to noise ratio $\rho_{S D}=\nu \gamma_{S D}, \rho_{S R}=\nu \gamma_{S R}, \rho_{R D}=$ $(1-\nu) \gamma_{R D}$.

We use a fixed threshold $s$ to switch between direct transmission and cooperation. In fact, when the source has good signal quality $\left(\gamma_{S D}>\gamma_{s}\right)$, only the direct link is used, otherwise, the transmission requires the assistance of the relay.

Now, the question is how the threshold value $s$ should be fixed. In order to determine the optimum threshold, we propose to trace the curves of capacity and BER (Bit Error Rate) for different values of $s$ as shown in figure 3 and figure 4 .

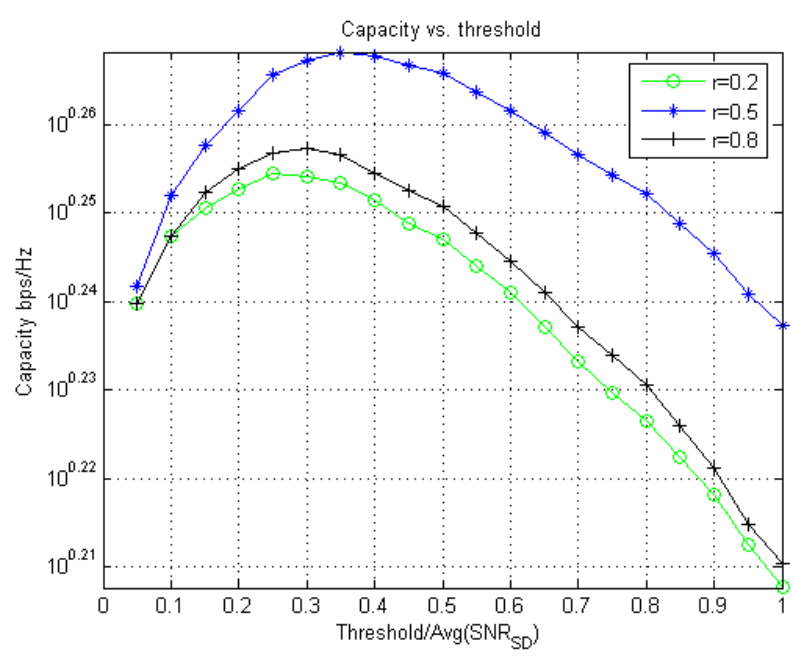

Fig. 3. Capacity for different threshold values

In this figure(Fig.3), we illustrate the capacity vs. the threshold $s$ that varies from 0.1 to 0.9 . For a cooperative power allocation $\nu=0.5$ (equal power at $\mathrm{S}$ and $\mathrm{D}$ ) and an average SNR of the direct link $\operatorname{rod} B=5 d B$,we can remark that the capacity grows until it achieves its peak and then decreases.

In the following we consider $r$ the relay location. The relay moves to be near the source $(r=0.2)$, in the middle $(r=0.5)$ or near the destination $(r=0.8)$.

For $r=0.2$, we can perceive that the capacity grows and its peak is achieved when $s=0.25$, where the corresponding capacity is $1.79 \mathrm{bps} / \mathrm{Hz}$.

For $r=0.5$, we can remark that the capacity increases until it achieves its peak when roughly $s=0.35$. In this case, the maximum achieved capacity is equal to $1.87 \mathrm{bps} / \mathrm{Hz}$.

For $r=0.8$, it is outstanding that the capacity enhances until it attains its top when $s=0.3$ with a value of $1.81 \mathrm{bps} / \mathrm{Hz}$ for the capacity, then it decreases.

We can deduce that the best values of the capacity are achieved when the relay is located in the middle between the source and the destination $(r=0.5)$, where $s=0.35$.

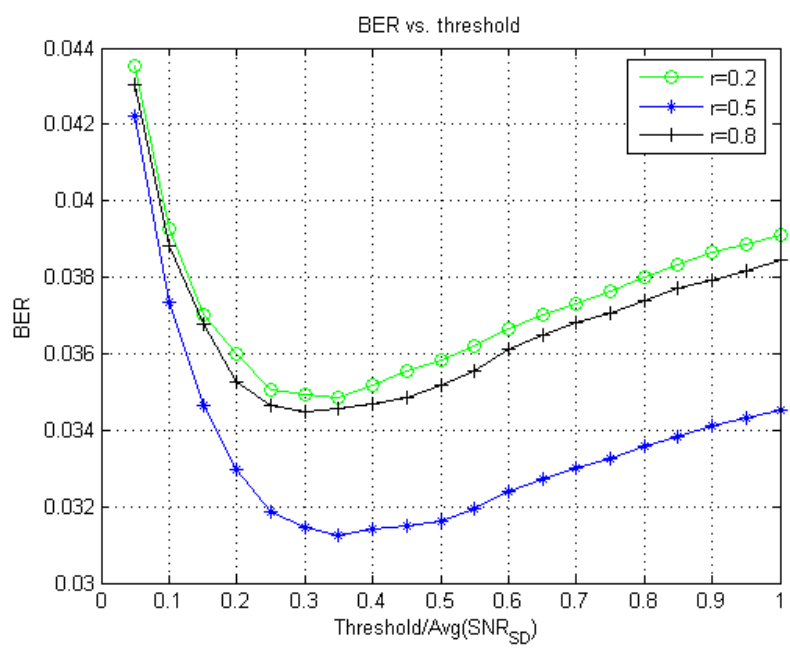

Fig. 4. BER for different threshold values

In figure (Fig.4), we show the BER vs. the threshold $s$ that varies from 0.1 to 0.9 . For $\nu=0.5$, and $\operatorname{rod} B=5 d B$, we can remark that the BER decreases until it achieves its minimum, then increases.

For $r=0.2$, we can perceive that the BER decreases until it reaches its minimum when $s=0.35$, the corresponding BER is $35 * 10^{-3}$.

For $r=0.5$, we can remark that the BER decreases promptly till it achieves its minimum when roughly $s=0.35$. In this case, it is equal to $31 * 10^{-3}$. After that, it increases.

For $r=0.8$, the BER minimum is achieved when $s=0.3$ with a value of $34 * 10^{-3}$.

We can deduce that the best values of BER are achieved when the relay is located in the middle between the source and the destination $(r=0.5)$, where $s=0.35$.

For the considered constellations, when the relay $\mathrm{R}$ is very close to the source $\mathrm{S}$ more power at the relay is required above as for $r=0.2$. When the relay $\mathrm{R}$ is so close to destination $\mathrm{D}$ as $r=0.8$, the source requires more power 
to send symbols to the relay. If $\mathrm{R}$ is in the middle region equal power shared between source and destination in the best choice. It is obvious that the best position of the relay is in the middle between the source and the destination because the system with equal power allocation will meet the performance of the optimal power control.

For example, if the path-loss exponent $\alpha=3$, and for equal power at S and D, $s_{\text {opt }}=0.35$ as shown in figure 3 and 4 .

In order to extract precise results, we construct the following comparative tables that present threshold variation according to rodB values for different relay's positions:

TABLE I

DIFFERENT VALUES OF CAPACITY AND THRESHOLD VARIATION FOR $r=0.2$

\begin{tabular}{|c|c|c|c|c|c|}
\hline rodB & 2 & 5 & 8 & 10 & 20 \\
\hline optimal threshold"s" & 0.3 & 0.25 & 0.25 & 0.15 & 0.08 \\
\hline Capacity & 1.20 & 1.79 & 2.52 & 3.00 & 5.94 \\
\hline
\end{tabular}

TABLE II

DIFFERENT VALUES OF CAPACITY AND THRESHOLD VARIATION FOR $r=0.5$

\begin{tabular}{|c|c|c|c|c|c|}
\hline rodB & 2 & 5 & 8 & 10 & 20 \\
\hline optimal threshold "s" & 0.4 & 0.35 & 0.3 & 0.25 & 0.09 \\
\hline Capacity & 1.25 & 1.87 & 2.56 & 3.05 & 5.96 \\
\hline
\end{tabular}

TABLE III

DIFFERENT VALUES OF CAPACITY AND THRESHOLD VARIATION FOR $r=0.8$

\begin{tabular}{|c|c|c|c|c|c|}
\hline rodB & 2 & 5 & 8 & 10 & 20 \\
\hline optimal threshold"s" & 0.3 & 0.3 & 0.25 & 0.2 & 0.08 \\
\hline Capacity & 1.20 & 1.81 & 2.51 & 3.00 & 5.94 \\
\hline
\end{tabular}

Observing the variation of the threshold values according to rodB, we see that for low average SNR of the direct link, the optimal threshold values are high:

Indeed, for $\operatorname{rod} B=5 d B$, first, when the relay is located close to the source, $r=0.2$ (table 1)the threshold value $s$ is equal to 0.25 , then, when the relay is in the middle, $r=0.5$ (table 2 ) the threshold value $s$ is equal to 0.35 and finally when the relay is near to the destination, $r=0.8$ (table 3 )the threshold value is equal to 0.3 .

However, for high average direct link SNR, the optimal threshold values are nearly equal to zero:

In fact, for $\operatorname{rod} B=20 \mathrm{~d} B$, first, in the case of $r=0.2$ (table 1) $s=0.08$, then, in the case of $r=0.5$ (table 2) $s=0.09$ and finally in the case of $r=0.8$ (table 3) $s=0.08$.

We can conclude from previous results that incremental relaying system is available only for low values of average direct link SNR, as in the opposite case, $s$ is very low and as a consequence the transmitted message doesn't pass through the relay and always follows the direct path. So, when the link between the source and the destination has good SNRs, and consequently there is not a need for relay assistance.

\section{CONClusion}

In this work, we have addressed the question of optimization of resource management in AF incremental and adaptive relaying systems. The joint optimisation of power assignment and the threshold of switching between direct and cooperative transmission is the main result.

\section{REFERENCES}

[1] A. Behnad, N. C. Beaulieu, and B. Maham, "Multi-hop amplify-andforward relaying on nakagami-0.5 fading channels," Wireless Communications Letters, IEEE, vol. 1, pp. 173 -176, june 2012.

[2] X. Zhang, W. Wang, and X. Ji, "Multiuser diversity in multiuser twohop cooperative relay wireless networks: System model and performance analysis," Vehicular Technology, IEEE Transactions on, vol. 58, pp. 1031 -1036 , february 2009.

[3] S. Ikki and M. Ahmed, "Performance analysis of cooperative diversity with incremental-best-relay technique over rayleigh fading channels," Communications, IEEE Transactions on, vol. 59, pp. 2152 -2161, august 2011.

[4] Y. Zhao, R. Adve, and T. Lim, "Improving amplify-and-forward relay networks: optimal power allocation versus selection," Wireless Communications, IEEE Transactions on, vol. 6, pp. 3114 -3123, august 2007.

[5] R. Hajji and N. Hamdi, "Optimizing of power allocation for two-hop df relaying systems," Electrotechnical conference, vol. 4, pp. $224-228$, Marth 2012.

[6] N. C. Beaulieu and J. Hui, "A closed-form expression for the outage probability of decode-and-forward relaying in dissimilar rayleigh fading channels," IEEE Commun. Letters, vol. 10, pp.813-815, vol. 13, pp. 687691, December 2006.

[7] P. J. S. H. A. Suraweera and J. Armstrong, "Outage probability of cooperative relay networks in nakagami-m fading channels," IEEE Commun. Letters, vol. 10, pp.834-836, vol. 7, pp. 687-691, December 2006.

[8] M. A. Nesrine Belhaj, Nouredine Hamdi, "Low-power minimum estimation and combining with adaptive modulation," International Conference on Information Sciences Signal Processing and their Applications ISSPA vol. 13, pp. 687-691, September 2005. 